Bull. Austral. Math. Soc.

$46 \mathrm{~A} 03,46 \mathrm{~A} 20,49 \mathrm{~K} 35$

VoL. $66(2002) \quad[43-56]$

\title{
A NEW MINIMAX THEOREM AND A PERTURBED JAMES'S THEOREM
}

\author{
M. Ruiz Galán and S. Simons
}

\begin{abstract}
The main result of this paper is a sufficient condition for the minimax relation to hold for the canonical bilinear form on $X \times Y$, where $X$ is a nonempty convex subset of a real locally convex space and $Y$ is a nonempty convex subset of its dual. Using the known "converse minimax theorem", this result leads easily to a nonlinear generalisation of James's ("sup") theorem. We give a brief discussion of the connections with the "sup-limsup theorem" and, in the appendix to the paper, we give a simple, direct proof (using Goldstine's theorem) of the converse minimax theorem referred to above, valid for the special case of a normed space.
\end{abstract}

\section{INTRODUCTION}

This paper is in two main parts. The first three sections give various result about bounded functions on an abstract set, while the last section is more functional-analytic in character.

The main functional-analytic result is Theorem 14, which contains a sufficient condition for the minimax relation to hold for the canonical bilinear form on $X \times Y$, where $X$ is a nonempty convex subset of a real locally convex space, $E$, and $Y$ is a nonempty convex subset of its dual, $E^{*}$. (The notation necessary for an understanding of Theorem 14 appears at the beginning of Section 4.) Using the known "converse minimax theorem", Theorem 14 leads easily to Theorem 16, a nonlinear generalisation of James's ("sup") theorem.

Theorem 14 depends on Theorem 13, which gives a sufficient condition for there to exist a set of functions, all of which fail to attain their maximum value on $X$. The statement that $\tilde{g} \in \operatorname{liminfsup}_{i} g_{i}$ means that $\liminf _{i} g_{i} \leqslant \tilde{g} \leqslant \limsup _{i} g_{i}$ on $X$-we describe such a function $\widetilde{g}$ as an "undetermined function". In the situation of Theorem 14, condition (14.1) ensures that one of these undetermined functions can be chosen to be the restriction to $X$ of an element of $E^{*}$, which is exactly what is needed for the proof of Theorem 14. All proofs of James's theorem also seem to need this undetermined function.

Received 11th December, 2001

Copyright Clearance Centre, Inc. Serial-fee code: 0004-9727/02 \$A2.00+0.00. 
Theorem 13 has two main components: a convexity argument specific to the canonical bilinear form and Theorem 10, which gives a sufficient condition (again, with an undetermined function) for there to exist a set of functions, all of which fail to attain their maximum value on a set $X$, but now $X$ is simply any nonempty set with no vector space structure.

Theorem 10 relies on a technique used by Pryce in his proof of James's theorem (see Lemma 9) and a strengthened form of an argument used in [8], (see Lemmas 4 and 5),

In Section 2, we digress a little to discuss the "sup-limsup theorem" proved in [8], and show how its proof contrasts with what we need to obtain our results on undetermined functions.

In the appendix to this paper, we give a simple, direct proof (using Goldstine's theorem) of the converse minimax theorem referred to above, valid for the special case when $E$ is a normed space.

\section{Preliminary Results}

Let $\mathcal{P}$ be the set of all real sequences $\left\{\lambda_{j}\right\}_{j \geqslant 1}$ such that, for all $j \geqslant 1, \lambda_{j} \geqslant 0$ and $\sum_{j=1}^{\infty} \lambda_{j}=1$. ("P " stands for "probability".) We first give an elementary property of $\mathcal{P}$.

LEMMA 1. Suppose that $\left\{\mu_{i}\right\}_{i \geqslant 1} \in \mathcal{P}$ and, for all $i \geqslant 1,\left\{\lambda_{i j}\right\}_{j \geqslant 1} \in \mathcal{P}$. For all $j \geqslant 1$, let $\nu_{j}:=\sum_{i=1}^{\infty} \mu_{i} \lambda_{i j} \geqslant 0$. Then $\left\{\nu_{j}\right\}_{j \geqslant 1} \in \mathcal{P}$.

PROOF: Since $\sum_{i=1}^{\infty} \sum_{j=1}^{\infty} \mu_{i} \lambda_{i j}=\sum_{i=1}^{\infty} \mu_{i} \sum_{j=1}^{\infty} \lambda_{i j}=\sum_{i=1}^{\infty} \mu_{i}=1$, from the double series theorem, $\sum_{j=1}^{\infty} \sum_{i=1}^{\infty} \mu_{i} \lambda_{i j}=1$, that is to say, $\sum_{j=1}^{\infty} \nu_{j}=1$.

Now let $H$ be a real sequentially complete Hausdorff locally convex space and $\left\{a_{j}\right\}_{j \geqslant 1}$ be a bounded sequence in $H$. If $\left\{\lambda_{j}\right\}_{j \geqslant 1} \in \mathcal{P}$ then $\sum_{j=1}^{\infty} \lambda_{j} a_{j}$ is defined in $H$ to be $\lim _{n \rightarrow \infty} \sum_{j=1}^{n} \lambda_{j} a_{j}$ (which exists by sequential completeness) and, whenever $p$ is a \[ n \rightarrow \infty \]
continuous seminorm on $H, p\left(\sum_{j=1}^{\infty} \lambda_{j} a_{j}\right) \leqslant \sum_{j=1}^{\infty} \lambda_{j} p\left(a_{j}\right)$. We write $\operatorname{co}_{\sigma}\left\{a_{j}: j \geqslant 1\right\}$ for
the set

$$
\left\{\sum_{j=1}^{\infty} \lambda_{j} a_{j}:\left\{\lambda_{j}\right\}_{j \geqslant 1} \in \mathcal{P}\right\}
$$

The operator $\mathrm{Co}_{\sigma}$ has a simple but important stability property, which is contained in the following lemma:

LEMma 2. Suppose that $\left\{a_{j}\right\}_{j \geqslant 1}$ is a bounded sequence in $H$ and, for all $i \geqslant 1$, 
$b_{i} \in \operatorname{co}_{\sigma}\left\{a_{j}: j \geqslant 1\right\}$. Then

$$
\operatorname{co}_{\sigma}\left\{b_{i}: i \geqslant 1\right\} \subset \operatorname{co}_{\sigma}\left\{a_{j}: j \geqslant 1\right\} .
$$

Proof: For all $i \geqslant 1$, let $\left\{\lambda_{i j}\right\}_{j \geqslant 1} \in \mathcal{P}$ and $b_{i}=\sum_{j=1}^{\infty} \lambda_{i j} a_{j}$. Let $c$ be an arbitrary element of $\operatorname{co}_{\sigma}\left\{b_{i}: i \geqslant 1\right\}$. Then there exists $\left\{\mu_{i}\right\}_{i \geqslant 1}^{j=1} \in \mathcal{P}$ such that $c=\sum_{i=1}^{\infty} \mu_{i} b_{i}$. Define $\left\{\nu_{j}\right\}_{j \geqslant 1} \in \mathcal{P}$ as in Lemma 1 . We shall show that

$$
c=\sum_{j=1}^{\infty} \nu_{j} a_{j} .
$$

This establishes that $c \in \operatorname{co}_{\sigma}\left\{a_{j}: j \geqslant 1\right\}$, which gives the required result since $c$ was an arbitrary element of $\operatorname{co}_{\sigma}\left\{b_{i}: i \geqslant 1\right\}$. Now, for all $n \geqslant 1$,

$$
\sum_{i=1}^{\infty} \sum_{j=1}^{\infty} \mu_{i} \lambda_{i j}=\sum_{i=1}^{n} \sum_{j=1}^{n} \mu_{i} \lambda_{i j}+\sum_{i=1}^{n} \sum_{j=n+1}^{\infty} \mu_{i} \lambda_{i j}+\sum_{i=n+1}^{\infty} \sum_{j=1}^{n} \mu_{i} \lambda_{i j}+\sum_{i=n+1}^{\infty} \sum_{j=n+1}^{\infty} \mu_{i} \lambda_{i j} .
$$
Since it follows from the double series theorem that $\sum_{i=1}^{n} \sum_{j=1}^{n} \mu_{i} \lambda_{i j} \rightarrow \sum_{i=1}^{\infty} \sum_{j=1}^{\infty} \mu_{i} \lambda_{i j}$ as
$n \rightarrow \infty$, we have

$$
\sum_{i=1}^{n} \sum_{j=n+1}^{\infty} \mu_{i} \lambda_{i j}+\sum_{i=n+1}^{\infty} \sum_{j=1}^{n} \mu_{i} \lambda_{i j}+\sum_{i=n+1}^{\infty} \sum_{j=n+1}^{\infty} \mu_{i} \lambda_{i j} \rightarrow 0 \text { as } n \rightarrow \infty .
$$

Now, for all $n \geqslant 1$,

$$
\begin{aligned}
\sum_{i=1}^{n} \mu_{i} b_{i}-\sum_{j=1}^{n} \nu_{j} a_{j} & =\sum_{i=1}^{n} \mu_{i}\left(\sum_{j=1}^{n} \lambda_{i j} a_{j}+\sum_{j=n+1}^{\infty} \lambda_{i j} a_{j}\right)-\sum_{j=1}^{n}\left(\sum_{i=1}^{n} \mu_{i} \lambda_{i j}+\sum_{i=n+1}^{\infty} \mu_{i} \lambda_{i j}\right) a_{j} \\
& =\sum_{i=1}^{n} \mu_{i} \sum_{j=n+1}^{\infty} \lambda_{i j} a_{j}-\sum_{j=1}^{n} \sum_{i=n+1}^{\infty} \mu_{i} \lambda_{i j} a_{j} .
\end{aligned}
$$

Thus $p\left(\sum_{i=1}^{n} \mu_{i} b_{i}-\sum_{j=1}^{n} \nu_{j} a_{j}\right) \leqslant \sum_{i=1}^{n} \sum_{j=n+1}^{\infty} \mu_{i} \lambda_{i j} p\left(a_{j}\right)+\sum_{j=1}^{n} \sum_{i=n+1}^{\infty} \mu_{i} \lambda_{i j} p\left(a_{j}\right)$ whenever $p$ is a continuous seminorm on $H$, and it follows from (2.2) and the fact that $\left\{a_{j}\right\}_{j \geqslant 1}$ is bounded that $\sum_{i=1}^{n} \mu_{i} b_{i}-\sum_{j=1}^{n} \nu_{j} a_{j} \rightarrow 0$ as $n \rightarrow \infty$. This gives (2.1), which completes the proof of Lemma 2.

Now let $X \neq \emptyset$. We are going to apply Lemma 2 in Lemma 4, Theorem 7 and Theorem 14, with $H$ the Banach space $\ell_{\infty}(X)$ of bounded real functions on $X$ with the supremum norm. Lemma 2 is more general than we need for this particular application. However, there are spaces in which Lemma 2 applies that are not Banach spaces - for 
instance, (when $X$ is infinite) the space $\mathbb{R}^{X}$ of all real functions on $X$ with the topology of pointwise convergence.

If $f \in \ell_{\infty}(X)$, we write $S_{X}(f):=\sup _{X} f$ and $\operatorname{argmax}_{X} f$ for $\{t \in X$ : $\left.f(t)=S_{X}(f)\right\}$. In what follows, $\sum_{i=1}^{0} \ldots$ is always interpreted to be 0.

Definition 3: Let $\left\{a_{j}\right\}_{j \geqslant 1}$ be a bounded sequence in $\ell_{\infty}(X)$. We say that $\left\{b_{i}\right\}_{i \geqslant 1}$ is a pseudo-subsequence of $\left\{a_{j}\right\}_{j} \geqslant 1$ if, for all $i \geqslant 1, b_{i} \in \operatorname{co}_{\sigma}\left\{a_{j}: j \geqslant i\right\}$.

LEMMA 4. Let $\left\{a_{j}\right\}_{j \geqslant 1}$ be a bounded sequence in $\ell_{\infty}(X)$ and $\rho \in(0,1)$.

(a) Let $\eta>0$. Then there exists a pseudo-subsequence $\left\{b_{i}\right\}_{i \geqslant 1}$ of $\left\{a_{j}\right\}_{j \geqslant 1}$ such that

$$
k \geqslant 0 \Longrightarrow S_{X}\left(\sum_{i=1}^{\infty} \rho^{i} b_{i}\right) \geqslant S_{X}\left(\sum_{i=1}^{k} \rho^{i} b_{i}\right)+\rho^{k}\left[S_{X}\left(\sum_{i=1}^{\infty} \rho^{i} b_{i}\right)-\eta\right] .
$$

(b) Let $B<\inf S_{X}\left(\operatorname{co}_{\sigma}\left\{a_{j}: j \geqslant 1\right\}\right)$. Then there exists a pseudo-subsequence $\left\{b_{i}\right\}_{i \geqslant 1}$ of $\left\{a_{j}\right\}_{j \geqslant 1}$ such that

$$
k \geqslant 0 \quad \Longrightarrow \quad S_{X}\left(\sum_{i=1}^{\infty} \rho^{i} b_{i}\right) \geqslant S_{X}\left(\sum_{i=1}^{k} \rho^{i} b_{i}\right)+B \sum_{i=k+1}^{\infty} \rho^{i} .
$$

Proof: (a) Suppose first that $m \geqslant 1$ and $b_{1}, \ldots, b_{m} \in \operatorname{co}_{\sigma}\left\{a_{j}: j \geqslant 1\right\}$. Then

$$
S_{X}\left(\sum_{i=1}^{m} \rho^{i} b_{i}\right) \geqslant-\left(\sum_{i=1}^{m} \rho^{i}\right) \sup _{i=1}^{m}\left\|b_{i}\right\| \geqslant-\left(\sum_{i=1}^{m} \rho^{i}\right) \sup _{j \geqslant 1}\left\|a_{j}\right\|>-\infty .
$$

For all $m \geqslant 1$, let $C_{m}:=\operatorname{co}_{\sigma}\left\{a_{j}: j \geqslant m\right\}$. Then we can choose $b_{m} \in C_{m}$ inductively so that

$$
S_{X}\left(\sum_{i=1}^{m-1} \rho^{i} b_{i}+\rho^{m} b_{m}\right) \leqslant \inf _{b \in C_{m}} S_{X}\left(\sum_{i=1}^{m-1} \rho^{i} b_{i}+\rho^{m} b\right)+\eta(\rho / 2)^{m} .
$$

(We note from (4.3) that $\inf _{b \in C_{m}} S_{X}\left(\sum_{i=1}^{m-1} \rho^{i} b_{i}+\rho^{m} b\right)>-\infty$.) Define $c:=\sum_{i=1}^{\infty} \rho^{i} b_{i}$ and, for all $m \geqslant 1, c_{m}:=\sum_{i=1}^{m} \rho^{i} b_{i}$ (so $c_{0}=0$ ). Then (4.4) gives

$$
\text { for all } m \geqslant 1, \quad S_{X}\left(c_{m}\right) \leqslant \inf _{b \in C_{m}} S_{X}\left(c_{m-1}+\rho^{m} b\right)+\eta(\rho / 2)^{m} \text {. }
$$

(4.1) is obvious when $k=0$. Now let $k \geqslant 1$ and $1 \leqslant m \leqslant k$. Then, from Lemma 2, $(1-\rho)\left(c-c_{m-1}\right) / \rho^{m}=\sum_{i=0}^{\infty}\left(\rho^{i}-\rho^{i+1}\right) b_{i+m} \in C_{m}$. Thus, from (4.5) and the sublinearity of $S_{X}$ on $\ell_{\infty}(X)$,

$$
\begin{aligned}
S_{X}\left(c_{m}\right) & \leqslant S_{X}\left(c_{m-1}+(1-\rho)\left(c-c_{m-1}\right)\right)+\eta(\rho / 2)^{m} \\
& =S_{X}\left((1-\rho) c+\rho c_{m-1}\right)+\eta(\rho / 2)^{m} \\
& \leqslant(1-\rho) S_{X}(c)+\rho S_{X}\left(c_{m-1}\right)+\eta(\rho / 2)^{m} .
\end{aligned}
$$


Dividing this by $\rho^{m}$, we obtain

$$
\left(1 / \rho^{m}-1 / \rho^{m-1}\right) S_{X}(c) \geqslant S_{X}\left(c_{m}\right) / \rho^{m}-S_{X}\left(c_{m-1}\right) / \rho^{m-1}-\eta / 2^{m}
$$

Adding up these inequalities for $m=1,2, \ldots, k$ (and noting that $c_{0}=0$ ) yields

$$
\left(1 / \rho^{k}-1\right) S_{X}(c) \geqslant S_{X}\left(c_{k}\right) / \rho^{k}-\eta
$$

which gives (4.1) on rearrangement.

(b) Let $\eta:=\sum_{i=1}^{\infty} \rho^{i}\left[\inf S_{X}\left(\operatorname{co}_{\sigma}\left\{a_{j}: j \geqslant 1\right\}\right)-B\right]>0$, and $\left\{b_{i}\right\}_{i \geqslant 1}$ be chosen as in (a) for this value of $\eta$. Lemma 2 gives

$$
S_{X}\left(\sum_{i=1}^{\infty} \rho^{i} b_{i}\right)-\eta \geqslant\left(\sum_{i=1}^{\infty} \rho^{i}\right) \inf S_{X}\left(\operatorname{co}_{\sigma}\left\{a_{j}: j \geqslant 1\right\}\right)-\eta=\sum_{i=1}^{\infty} \rho^{i} B
$$

and (4.2) follows by substituting this into (4.1).

LEMMA 5. Let $\left\{b_{i}\right\}_{i \geqslant 1}$ be a bounded sequence in $\ell_{\infty}(X)$ satisfying (4.2) for some $\rho \in(0,1)$ and $B \in \mathbb{R}$. Let $M:=\sup _{i \geqslant 1}\left\|b_{i}\right\| \in \mathbb{R}$. Then

$$
p \in \operatorname{argmax}_{X}\left(\sum_{i=1}^{\infty} \rho^{i} b_{i}\right) \Longrightarrow \inf _{k \geqslant 1} b_{k}(p) \geqslant \frac{B-\rho M}{1-\rho}
$$

Proof: Let $p \in \operatorname{argmax}_{X}\left(\sum_{i=1}^{\infty} \rho^{i} b_{i}\right)$. Then, from (4.2),

$$
k \geqslant 0 \Longrightarrow \sum_{i=1}^{\infty} \rho^{i} b_{i}(p) \geqslant \sum_{i=1}^{k} \rho^{i} b_{i}(p)+B \sum_{i=k+1}^{\infty} \rho^{i}
$$

from which

$$
k \geqslant 1 \Longrightarrow \sum_{i=k}^{\infty} \rho^{i-k} b_{i}(p) \geqslant B \sum_{i=0}^{\infty} \rho^{i}
$$

and so

$$
k \geqslant 1 \Longrightarrow b_{k}(p) \geqslant B \sum_{i=0}^{\infty} \rho^{i}-\sum_{i=1}^{\infty} \rho^{i} M
$$

which gives (5.1).

LEMMA 6. Let $\left\{a_{j}\right\}_{j \geqslant 1}$ be a bounded sequence in $\ell_{\infty}(X)$ and $\left\{b_{i}\right\}_{i \geqslant 1}$ be a pseudo-subsequence of $\left\{a_{j}\right\}_{j \geqslant 1}$. Then

$$
\liminf a_{j} \leqslant \liminf _{i} b_{i} \leqslant \limsup _{i} b_{i} \leqslant \limsup _{j} a_{j} \text { on } X .
$$


This holds, in particular, if $\left\{b_{i}\right\}_{i \geqslant 1}$ is a subsequence of $\left\{a_{j}\right\}_{j \geqslant 1}$.

Proof: Let $i \geqslant 1$. Then there exists $\left\{\lambda_{j}\right\}_{j \geqslant i} \in \mathcal{P}$ such that $b_{i}=\sum_{j=i}^{\infty} \lambda_{j} a_{j}$. It follows that $b_{i} \leqslant \sup _{j \geqslant i} a_{j}$ on $X$, from which

$\lim \sup _{i} b_{i} \leqslant \limsup \sup _{j} \operatorname{sun}_{j}=\limsup _{j} a_{j}$ on $X$

The proof that $\liminf _{j} a_{j} \leqslant \liminf _{i} b_{i}$ on $X$ is similar.

\section{ThE SUP-LIMSUP THEOREM}

In this short section, we discuss the "sup-limsup theorem", first proved in a Banach space context in [8]. (See also Oja, [4, Theorem 2.2, p.2807-2808].) Though it is a digression from our main theme, it provides an interesting comparison. Theorem 7 uses the technique of Lemma 5 , organised in a slightly different way. It also uses Lemma 4(a) with $\rho=1 / 2$. This contrasts with the situation in Corollary 8 , in which we take $\rho$ to be small. The precise place where we need this is the statement "since $\inf _{k \geqslant 1} g_{k} \leqslant \tilde{g}$ on $X$ " towards the end of the proof of Corollary 8. (It is not true that $\sup g_{k} \leqslant \tilde{g}$ on $X$.) Corollary 8 leads to Theorem 10, the result on "undetermined functions", that leads in turn to our main result, Theorem 14.

This is a good place to mention the paper [2] by Godefroy, which contains many other applications of similar ideas to Banach spaces, as well as further references.

THEOREM 7. Let $\left\{f_{k}\right\}_{k \geqslant 1}$ be a bounded sequence in $\ell_{\infty}(X)$ and suppose that $P$ is a "peak set" for $\operatorname{co}_{\sigma}\left\{f_{k}: k \geqslant 1\right\}$, that is, for all $f \in \operatorname{co}_{\sigma}\left\{f_{k}: k \geqslant 1\right\}, P \cap \operatorname{argmax}_{X} f$ $\neq \emptyset$. Then

$$
S_{P}\left(\lim \sup _{k} f_{k}\right)=S_{X}\left(\lim \sup _{k} f_{k}\right)
$$

Proof: It is obvious that $S_{P}\left(\limsup f_{k}\right) \leqslant S_{X}\left(\limsup f_{k} f_{k}\right)$, so we now prove " $\geqslant "$. Let $\eta>0$. We first choose $x \in X$ so that $\limsup \sup _{k} f_{k}(x)>S_{X}\left(\limsup f_{k} f_{k}\right)-\eta$, and then choose a subsequence $\left\{a_{j}\right\}_{j \geqslant 1}$ of $\left\{f_{k}\right\}_{k \geqslant 1}$ such that $\inf _{j \geqslant 1} a_{j}(x) \geqslant S_{X}\left(\limsup f_{k} f_{k}\right)$ $-\eta$. It follows that

$$
S_{X}\left(\inf _{j \geqslant 1} a_{j}\right) \geqslant S_{X}\left(\lim \sup _{k} f_{k}\right)-\eta
$$

From Lemma 4 (a) with $\rho=1 / 2$, there exists a pseudo-subsequence $\left\{b_{i}\right\}_{i \geqslant 1}$ of $\left\{a_{j}\right\}_{j \geqslant 1}$ such that,

$$
k \geqslant 0 \Longrightarrow S_{X}\left(\sum_{i=1}^{\infty} b_{i} / 2^{i}\right) \geqslant S_{X}\left(\sum_{i=1}^{k} b_{i} / 2^{i}\right)+\left(S_{X}\left(\sum_{i=1}^{\infty} b_{i} / 2^{i}\right)-\eta\right) / 2^{k}
$$


Arguing as in Lemma $6, \sum_{i=1}^{\infty} b_{i} / 2^{i} \geqslant \inf _{i \geqslant 1} b_{i} \geqslant \inf _{j \geqslant 1} a_{j}$ on $X$ so, from (7.1),

$$
k \geqslant 0 \Longrightarrow S_{X}\left(\sum_{i=1}^{\infty} b_{i} / 2^{i}\right) \geqslant S_{X}\left(\sum_{i=1}^{k} b_{i} / 2^{i}\right)+\left(S_{X}\left(\limsup \sup _{k}\right)-2 \eta\right) / 2^{k}
$$

By hypothesis and Lemma 2, there exists $p \in P \cap \operatorname{argmax}_{X}\left(\sum_{i=1}^{\infty} b_{i} / 2^{i}\right)$. Consequently

$$
\begin{aligned}
k \geqslant 0 & \Longrightarrow \sum_{i=1}^{\infty} b_{i}(p) / 2^{i} \geqslant \sum_{i=1}^{k} b_{i}(p) / 2^{i}+\left(S_{X}\left(\limsup \sup _{k} f_{k}\right)-2 \eta\right) / 2^{k} \\
& \Longrightarrow \quad \sum_{i=k+1}^{\infty} b_{i}(p) / 2^{i} \geqslant\left(S_{X}\left(\lim \sup _{k} f_{k}\right)-2 \eta\right) / 2^{k} \\
& \Longrightarrow \sum_{i=1}^{\infty} b_{i+k}(p) / 2^{i} \geqslant S_{X}\left(\limsup \sup _{k} f_{k}\right)-2 \eta \\
& \Longrightarrow \sup _{i>k} b_{i}(p) \geqslant S_{X}\left(\lim \sup _{k} f_{k}\right)-2 \eta
\end{aligned}
$$

Letting $k \rightarrow \infty$, we obtain that $\limsup _{i} b_{i}(p) \geqslant S_{X}\left(\limsup f_{k}\right)-2 \eta$. Two application of Lemma 6 now give $\lim \sup _{k} f_{k} \geqslant \lim \sup _{j} a_{j} \geqslant \limsup b_{i} b_{i}$ on $X$, and so $\lim \sup _{j} f_{j}(p) \geqslant S_{X}\left(\lim \sup _{k} f_{k}\right)-2 \eta$. The result follows since $\eta>0$ is arbitrary.

\section{THE TECHNIQUE OF THE UNDETERMINED FUNCTION}

If $\left\{g_{i}\right\}_{i \geqslant 1}$ is a bounded sequence in $\ell_{\infty}(X)$, we write $\operatorname{liminfsup}_{i} g_{i}$ for the set

$$
\left\{\tilde{g} \in \ell_{\infty}(X): \liminf _{i} g_{i} \leqslant \tilde{g} \leqslant \lim \sup _{i} g_{i} \text { on } X\right\} .
$$

COROLlary 8. Let $\varphi \in \ell_{\infty}(X)$ with $\varphi \geqslant 0$ on $X,\left\{h_{j}\right\}_{j \geqslant 1}$ be a bounded sequence in $\ell_{\infty}(X), A>0$ and suppose

$$
S_{X}\left(h_{0}-\lim \sup _{j} h_{j}-\varphi\right)=S_{X}\left(h_{0}-\liminf _{j} h_{j}-\varphi\right) \geqslant A
$$

for all $h_{0} \in \operatorname{co}_{\sigma}\left\{h_{j}: j \geqslant 1\right\}$. Then there exist a pseudo-subsequence $\left\{g_{i}\right\}_{i \geqslant 1}$ of $\left\{h_{j}\right\}_{j \geqslant 1}$ and $g_{0} \in \operatorname{co}_{\sigma}\left\{g_{i}: i \geqslant 1\right\}$ such that

$$
\tilde{g} \in \operatorname{liminfsup}_{\boldsymbol{i}} g_{i} \quad \Longrightarrow \quad \operatorname{argmax}_{X}\left(g_{0}-\tilde{g}-\varphi\right)=\emptyset .
$$

Proof: Let $N:=\sup _{n \geqslant 1}\left\|h_{n}\right\|$ and $\rho \in(0,1)$ be so small that $(2 N+\|\varphi\|+1) \rho$ $<A$. Let $\underline{h}:=\liminf _{j} h_{j} \in \ell_{\infty}(X), \bar{h}:=\limsup _{j} h_{j} \in \ell_{\infty}(X)$ and, for all $j \geqslant 1$, 
$a_{j}:=h_{j}-\underline{h}-\varphi$, so $\sup _{j \geqslant 1}\left\|a_{j}\right\| \leqslant 2 N+\|\varphi\|<\infty$. We now apply Lemma 4(b) with $B:=(2 N+\|\dot{\varphi}\|+1) \rho$. It follows that there exists a pseudo-subsequence $\left\{g_{i}\right\}_{i \geqslant 1}$ of $\left\{h_{j}\right\}_{j \geqslant 1}$ such that

$$
k \geqslant 0 \Longrightarrow S_{X}\left(\sum_{i=1}^{\infty} \rho^{i}\left(g_{i}-\underline{h}-\varphi\right)\right) \geqslant S_{X}\left(\sum_{i=1}^{k} \rho^{i}\left(g_{i}-\underline{h}-\varphi\right)\right)+B \sum_{i=k+1}^{\infty} \rho^{i}
$$

Let $\tilde{g} \in \operatorname{liminfsup}_{i} g_{i}$. We have from Lemma 6 that $\tilde{g} \in \operatorname{liminfsup}_{j} h_{j}$ and so the equality part of (8.1) gives us that

$$
k \geqslant 0 \Longrightarrow S_{X}\left(\sum_{i=1}^{\infty} \rho^{i}\left(g_{i}-\tilde{g}-\varphi\right)\right) \geqslant S_{X}\left(\sum_{i=1}^{k} \rho^{i}\left(g_{i}-\tilde{g}-\varphi\right)\right)+B \sum_{i=k+1}^{\infty} \rho^{i}
$$

For all $i \geqslant 1$, let $b_{i}:=g_{i}-\tilde{g}-\varphi$. Now $\sup _{i \geqslant 1}\left\|b_{i}\right\| \leqslant 2 N+\|\varphi\|<\infty$, and so we can apply Lemma 5. It follows that if $p \in \operatorname{argmax}_{X}\left(\sum_{i=1}^{\infty} \rho^{i}\left(g_{i}-\tilde{g}-\varphi\right)\right)$ then

$$
\inf _{k \geqslant 1}\left(g_{k}-\tilde{g}-\varphi\right)(p) \geqslant \frac{(2 N+\|\varphi\|+1) \rho-\rho(2 N+\|\varphi\|)}{1-\rho}=\frac{\rho}{1-\rho}>0,
$$

which is impossible, since $\inf _{k \geqslant 1} g_{k} \leqslant \tilde{g}$ on $X$ and $\varphi(p) \geqslant 0$. Consequently,

$$
\operatorname{argmax}_{X}\left(\sum_{i=1}^{\infty} \rho^{i}\left(g_{i}-\widetilde{g}-\varphi\right)\right)=\emptyset
$$

and the required result follows with $g_{0}:=\sum_{i=1}^{\infty} \rho^{i} g_{i} / \sum_{i=1}^{\infty} \rho^{i}$.

The proof of Lemma 9 below is based on a technique used by Pryce in his proof ([5]) of James's theorem.

Lemma 9.

(a) Let $\left\{a_{j}\right\}_{j \geqslant 1}$ be a bounded sequence in $\ell_{\infty}(X)$ and $\varepsilon>0$. Then there exists a subsequence $\left\{b_{j}\right\}_{j \geqslant 1}$ of $\left\{a_{j}\right\}_{j \geqslant 1}$ such that

$$
S_{X}\left(\liminf b_{j}\right) \geqslant S_{X}\left(\lim \sup _{j} a_{j}\right)-\varepsilon
$$

(b) Let $\left\{a_{j}\right\}_{j \geqslant 1}$ be a bounded sequence in $\ell_{\infty}(X)$. Then there exists a subsequence $\left\{b_{j}\right\}_{j \geqslant 1}$ of $\left\{a_{j}\right\}_{j \geqslant 1}$ such that

$$
S_{X}\left(\liminf b_{j}\right)=S_{X}\left(\limsup \sup _{j} b_{j}\right)
$$


(c) Let $\varphi \in \ell_{\infty}(X)$ and $\varphi \geqslant 0$ on $X$. Let $\left\{f_{j}\right\}_{j \geqslant 1}$ be a bounded sequence in $\ell_{\infty}(X)$. Then there exists a subsequence $\left\{h_{j}\right\}_{j \geqslant 1}$ of $\left\{f_{j}\right\}_{j \geqslant 1}$ such that

$$
S_{X}\left(h_{0}-\lim \sup _{j} h_{j}-\varphi\right)=S_{X}\left(h_{0}-\liminf _{j} h_{j}-\varphi\right)
$$

for all $h_{0} \in \operatorname{co}_{\sigma}\left\{h_{j}: j \geqslant 1\right\}$.

Proof: (a) We first choose $x \in X$ so that

$$
\lim \sup _{j} a_{j}(x) \geqslant S_{X}\left(\limsup a_{j} a_{j}\right)-\varepsilon
$$

and then choose a subsequence $\left\{b_{j}\right\}_{j \geqslant 1}$ of $\left\{a_{j}\right\}_{j \geqslant 1}$ so that

$$
\lim _{j} b_{j}(x)=\limsup _{j} a_{j}(x)-\text { hence } \liminf _{j} b_{j}(x) \geqslant S_{X}\left(\limsup _{j} a_{j}\right)-\varepsilon .
$$

This gives (9.1), and (a) follows immediately.

(b) For all $j \geqslant 1$, let $b_{j}^{(0)}:=a_{j}$. From (a), for all $m \geqslant 1$, we can define inductively a subsequence $\left\{b_{j}^{(m)}\right\}_{j \geqslant 1}$ of $\left\{b_{j}^{(m-1)}\right\}_{j \geqslant 1}$ so that

$$
S_{X}\left(\liminf _{j} b_{j}^{(m)}\right) \geqslant S_{X}\left(\limsup \sup _{j}^{(m-1)}\right)-1 / m .
$$

From the diagonal argument, there exists a bounded sequence $\left\{b_{j}\right\}_{j \geqslant 1}$ in $\ell_{\infty}(X)$ such that, for all $m \geqslant 1,\left\{b_{j}\right\}_{j \geqslant m}$ is a subsequence of $\left\{b_{j}^{(m)}\right\}_{j \geqslant 1}$, and (9.2) now follows from (9.4) by using Lemma 6 and letting $m \rightarrow \infty$.

(c) Since the set $\operatorname{co}_{\sigma}\left\{f_{j}: j \geqslant 1\right\}$ is norm-separable, we can choose $\left\{d_{m}: m \geqslant 1\right\}$ to be norm-dense in $\operatorname{co}_{\sigma}\left\{f_{j}: j \geqslant 1\right\}$. For all $j \geqslant 1$, let $h_{j}^{(0)}:=f_{j}$. Using (b) with $a_{j}:=d_{m}-h_{j}^{(m-1)}-\varphi$, for all $m \geqslant 1$, we can find a subsequence $\left\{h_{j}^{(m)}\right\}_{j \geqslant 1}$ of $\left\{h_{j}^{(m-1)}\right\}_{j \geqslant 1}$ inductively so that

$$
S_{X}\left(d_{m}-\lim \sup _{j} h_{j}^{(m)}-\varphi\right)=S_{X}\left(d_{m}-\liminf h_{j}^{(m)}-\varphi\right) .
$$

From the diagonal argument, there exists a bounded sequence $\left\{h_{j}\right\}_{j \geqslant 1}$ in $\ell_{\infty}(X)$ such that, for all $m \geqslant 1,\left\{h_{j}\right\}_{j \geqslant m}$ is a subsequence of $\left\{h_{j}^{(m)}\right\}_{j \geqslant 1}$. Then, from Lemma 6 and (9.5),

$$
\text { for all } m \geqslant 1, \quad S_{X}\left(d_{m}-\lim \sup _{j} h_{j}-\varphi\right)=S_{X}\left(d_{m}-\liminf _{j} h_{j}-\varphi\right) .
$$

(9.3) now follows since $S_{X}(\cdot-\varphi)$ is norm-continuous and $\left\{d_{m}: m \geqslant 1\right\}$ is normdense in the set $\operatorname{co}_{\sigma}\left\{f_{j}: j \geqslant 1\right\}$, and so certainly norm-dense in the (sub)set $\operatorname{co}_{\sigma}\left\{h_{j}: j \geqslant 1\right\}$. 
TheOREM 10. Let $\varphi \in \ell_{\infty}(X)$ and $\varphi \geqslant 0$ on $X$. Let $\left\{f_{j}\right\}_{j \geqslant 1}$ be a bounded sequence in $\ell_{\infty}(X), A>0$ and

$$
S_{X}\left(f_{0}-\lim \sup _{j} f_{j}-\varphi\right) \geqslant A
$$

for all $f_{0} \in \operatorname{co}_{\sigma}\left\{f_{j}: j \geqslant 1\right\}$. Then there exist a pseudo-subsequence $\left\{g_{i}\right\}_{i \geqslant 1}$ of $\left\{f_{j}\right\}_{j \geqslant 1}$ and $g_{0} \in \operatorname{co}_{\sigma}\left\{g_{i}: i \geqslant 1\right\}$ such that

$$
\operatorname{argmax}_{X}\left(g_{0}-\tilde{g}-\varphi\right)=\emptyset \text { for all } \tilde{g} \in \operatorname{liminfsup}_{i} g_{i}
$$

Proof: From Lemma 9(c), there exists a subsequence $\left\{h_{j}\right\}_{j \geqslant 1}$ of $\left\{f_{j}\right\}_{j \geqslant 1}$ satisfying (9.3) for all $h_{0} \in \operatorname{co}_{\sigma}\left\{h_{j}: j \geqslant 1\right\}$. Since $\operatorname{co}_{\sigma}\left\{h_{j}: j \geqslant 1\right\} \subset \operatorname{co}_{\sigma}\left\{f_{j}: j \geqslant 1\right\}$, (8.1) follows by combining this with Lemma 6 and (10.1). The result now follows from Corollary 8.

\section{A MiNIMAX THEOREM THAT IMPLIES A NONLINEAR VERSION OF JAMES'S THEOREM}

For the rest of this paper, we shall suppose that $E$ is a real locally convex space with dual $E^{*}$ and $\langle\cdot, \cdot\rangle$ is the canonical bilinear form on $E \times E^{*}$. We shall suppose also that $X$ is a nonempty convex subset of $E$ and $Y$ is a nonempty convex subset of $E^{*}$ such that $\langle\cdot, \cdot\rangle$ is bounded on $X \times Y$. We write " $\delta$ " as an alias for $\langle\cdot, \cdot\rangle$, so " $\sup _{X} \inf _{Y} \delta$ " stands for $\sup _{x \in X} \inf _{x^{*} \in Y}\left\langle x, x^{*}\right\rangle$ and "inf $\sup _{X} \delta^{\prime}$ "stands for $\inf _{x^{*} \in Y} \sup _{x \in X}\left\langle x, x^{*}\right\rangle$. Then, as is well known, $\sup _{X} \inf _{Y} \delta \leqslant \inf _{Y} \sup _{X} \delta$. We write $\operatorname{dgap}(X, Y):=\inf _{Y} \sup _{X} \delta-\sup _{X} \inf _{Y} \delta$. "dgap" stands for "duality gap". However, we should caution the reader that some authors use the phrase "duality gap" for the interval $\left[\sup _{X} \inf _{Y} \delta\right.$, inf $\left.\sup _{X} \delta\right]$. If $\varphi \in \ell_{\infty}(X)$, we write $\operatorname{osc}_{X} \varphi$ for the "oscillation" of $\varphi$ on $X$, defined by $\operatorname{osc}_{X} \varphi:=\sup _{X} \varphi-\inf { }_{X} \varphi$.

We shall need a fact about convex functions for our analysis. We could use a minimax theorem for this, but it is somewhat more direct to use the following result (see [9, Lemma 2.1, p.15]), which can also be deduced from Fan-Glicksberg-Hoffman, [1, Theorem 1, p.618], after some simple transformations.

LEMMA 11. Let $C$ be a nonempty convex subset of a vector space and $f_{1}, \ldots, f_{n}$ be concave real functions on $C$. Then there exist $\lambda_{1}, \ldots, \lambda_{n} \geqslant 0$ such that $\lambda_{1}+\cdots+$ $\lambda_{n}=1$ and

$$
\sup _{C}\left[f_{1} \wedge \cdots \wedge f_{n}\right]=\sup _{C}\left[\lambda_{1} f_{1}+\cdots+\lambda_{n} f_{n}\right]
$$

\section{LEMMA 12 .}

(a) Suppose that $\inf _{Y} \sup _{X} \delta>\beta$. Then, for all $x_{1}^{*}, \ldots, x_{n}^{*} \in Y$, there exists $x \in X$ such that $\left\langle x, x_{1}^{*}\right\rangle \wedge \cdots \wedge\left\langle x, x_{n}^{*}\right\rangle>\beta$.

(b) Suppose that $\sup _{X} \inf _{Y} \delta<\alpha$. Then, for all $x_{1}, \ldots, x_{n} \in X$, there exists $x^{*} \in Y$ such that $\left\langle x_{1}, x^{*}\right\rangle \vee \cdots \vee\left\langle x_{n}, x^{*}\right\rangle<\alpha$. 
Proof: From Lemma 11, there exist $\lambda_{1}, \ldots, \lambda_{n} \geqslant 0$ such that $\lambda_{1}+\cdots+\lambda_{n}=1$ and

$$
\begin{aligned}
\sup _{x \in X}\left[\left\langle x, x_{1}^{*}\right\rangle \wedge \cdots \wedge\left\langle x, x_{n}^{*}\right\rangle\right] & =\sup _{x \in X}\left[\lambda_{1}\left\langle x, x_{1}^{*}\right\rangle+\cdots+\lambda_{n}\left\langle x, x_{n}^{*}\right\rangle\right] \\
& \geqslant \sup _{x \in X}\left\langle x, \lambda_{1} x_{1}^{*}+\cdots+\lambda_{n} x_{n}^{*}\right\rangle>\beta,
\end{aligned}
$$

since $\lambda_{1} x_{1}^{*}+\cdots+\lambda_{n} x_{n}^{*} \in Y$. This completes the proof of (a), and the proof of (b) is similar.

THEOREM 13. Suppose that $\varphi \in \ell_{\infty}(X)$ and $\operatorname{osc}_{X} \varphi<\operatorname{dgap}(X, Y)$. Then there exist a sequence $\left\{x_{j}^{*}\right\}_{j \geqslant 1}$ in $Y$, a pseudo-subsequence $\left\{g_{i}\right\}_{i \geqslant 1}$ of $\left\{\left.x_{j}^{*}\right|_{X}\right\}_{j \geqslant 1}$ in $\ell_{\infty}(X)$ and $g_{0} \in \operatorname{co}_{\sigma}\left\{g_{i}: i \geqslant 1\right\}$ such that

$$
\operatorname{argmax}_{X}\left(g_{0}-\tilde{g}-\varphi\right)=\emptyset, \text { for all } \tilde{g} \in \operatorname{liminfsup}_{i} g_{i} \text {. }
$$

Proof: Since $\operatorname{osc}_{X} \varphi$ and (13.1) are unaffected by adding a constant to $\varphi$, we can and shall suppose that $\inf _{X} \varphi=0$, so $\varphi \geqslant 0$ on $X$ and also $\operatorname{osc}_{X} \varphi=S_{X}(\varphi)$. Let $M:=\sup |\langle X, Y\rangle|$, and choose $\alpha>\sup _{X} \inf _{Y} \delta$ and $\beta<\inf _{Y} \sup _{X} \delta$ so that $\beta-\alpha>\operatorname{osc}_{X} \varphi$. Let $x_{1}^{*}$ be an arbitrary element of $Y$. Then, using (a) and (b) of Lemma 12 alternately, we can find $x_{1} \in X, x_{2}^{*} \in Y, x_{2} \in X, \ldots$ so that

$$
\left\langle x_{n}, x_{1}^{*}\right\rangle \wedge \cdots \wedge\left\langle x_{n}, x_{n}^{*}\right\rangle>\beta \text { and }\left\langle x_{1}, x_{n+1}^{*}\right\rangle \vee \cdots \vee\left\langle x_{n}, x_{n+1}^{*}\right\rangle<\alpha .
$$

Write $f_{j}:=\left.x_{j}^{*}\right|_{X} \in \ell_{\infty}(X)$. Then $j \leqslant n \Longrightarrow f_{j}\left(x_{n}\right)>\beta$ and $n<j \Longrightarrow f_{j}\left(x_{n}\right)<\alpha$. It follows that, for all $n \geqslant 1, \limsup f_{j}\left(x_{n}\right) \leqslant \alpha$. On the other hand, if $f_{0} \in \operatorname{co}_{\sigma}\left\{f_{j}: j \geqslant 1\right\}$, then there exists a sequence $\left\{\lambda_{j}\right\}_{j \geqslant 1}$ of elements of $[0,1]$ such that $\sum_{j=1}^{\infty} \lambda_{j}=1$ and $f_{0}=\sum_{j=1}^{\infty} \lambda_{j} f_{j}$. Thus, for all $n \geqslant 1$,

$$
\begin{aligned}
f_{0}\left(x_{n}\right) & =\sum_{j=1}^{\infty} \lambda_{j} f_{j}\left(x_{n}\right)=\sum_{j=1}^{n} \lambda_{j} f_{j}\left(x_{n}\right)+\sum_{j=n+1}^{\infty} \lambda_{j} f_{j}\left(x_{n}\right) \\
& \geqslant \sum_{j=1}^{n} \lambda_{j} \beta-\sum_{j=n+1}^{\infty} \lambda_{j} M=\sum_{j=1}^{\infty} \lambda_{j} \beta-\sum_{j=n+1}^{\infty} \lambda_{j} \beta-\sum_{j=n+1}^{\infty} \lambda_{j} M \\
& =\beta-\sum_{j=n+1}^{\infty} \lambda_{j}(\beta+M) .
\end{aligned}
$$

Thus $S_{X}\left(f_{0}-\lim \sup _{j} f_{j}\right) \geqslant f_{0}\left(x_{n}\right)-\lim \sup _{j} f_{j}\left(x_{n}\right) \geqslant \beta-\alpha-\sum_{j=n+1}^{\infty} \lambda_{j}(\beta+M)$. If we now let $n \rightarrow \infty$, we obtain $S_{X}\left(f_{0}-\lim \sup _{j} f_{j}\right) \geqslant \beta-\alpha$, and the result follows from Theorem 10 since $S_{X}\left(f_{0}-\lim \sup _{j} f_{j}-\varphi\right) \geqslant \beta-\alpha-S_{X}(\varphi)>\operatorname{osc}_{X} \varphi-S_{X}(\varphi)=0$.

We now come to our main result, a minimax theorem generalising [7, Theorem 14 , p.715-6]. 
THEOREM 14. Suppose that every sequence in $Y$ has a $w\left(E^{*}, X\right)$-cluster point in $E^{*}$

and there exists $\psi \in \ell_{\infty}(X)$ such that,

$$
\text { for all } x^{*} \in E^{*}, \quad \operatorname{argmax}_{X}\left(\left.x^{*}\right|_{X}-\psi\right) \neq \emptyset .
$$

Then

$$
\inf _{Y} \sup _{X} \delta=\sup _{X} \inf _{Y} \delta
$$

Proof: If (14.3) fails then $\operatorname{dgap}(X, Y)>0$, so we can choose $\rho>0$ so that

$$
\operatorname{dgap}(X, Y)>\rho \operatorname{osc}_{X} \psi=\operatorname{osc}_{X} \rho \psi
$$

From Theorem 13, we can find a sequence $\left\{x_{j}^{*}\right\}_{j \geqslant 1}$ in $Y$, a pseudo-subsequence $\left\{g_{i}\right\}_{i \geqslant 1}$ of $\left\{\left.x_{j}^{*}\right|_{X}\right\}_{j \geqslant 1}$ in $\ell_{\infty}(X)$ and $g_{0} \in \operatorname{co}_{\sigma}\left\{g_{i}: i \geqslant 1\right\}$ such that

$$
\operatorname{argmax}_{X}\left(g_{0}-\tilde{g}-\rho \psi\right)=\emptyset, \text { for all } \tilde{g} \in \operatorname{liminfsup}_{i} g_{i}
$$

Lemma 2 implies that $g_{0} \in \operatorname{co}_{\sigma}\left\{\left.x_{j}^{*}\right|_{X}: j \geqslant 1\right\}$. Thus there exists $\left\{\lambda_{j}\right\}_{j \geqslant 1} \in \mathcal{P}$ such that $g_{0}=\left.\sum_{j=1}^{\infty} \lambda_{j} x_{j}^{*}\right|_{X}$ in $\ell_{\infty}(X)$. Fix $n_{0} \geqslant 1$ so that $\lambda_{n_{0}}>0$. For $n \geqslant n_{0}$, let

$$
y_{n}^{*}:=\sum_{j=1}^{n} \lambda_{j} x_{j}^{*} / \sum_{j=1}^{n} \lambda_{j} \in Y .
$$

From (14.1), $\left\{y_{n}^{*}\right\}_{n \geqslant n_{0}}$ has a $w\left(E^{*}, X\right)$-cluster point $y^{*} \in E^{*}$. Since $\left.y_{n}^{*}\right|_{X} \rightarrow g_{0}$ in $\ell_{\infty}(X)$, it follows that $g_{0}=\left.y^{*}\right|_{X}$. Now suppose that $i \geqslant 1$ and examine $g_{i}$. The argument above shows that there exists $z_{i}^{*} \in Y$ such that $\left\|\left.z_{i}^{*}\right|_{X}-g_{i}\right\| \leqslant 1 / i$ and, from (14.1), $\left\{z_{i}^{*}\right\}_{i \geqslant 1}$ has a $w\left(E^{*}, X\right)$-cluster point $z^{*} \in E^{*}$. It follows from all this that

$$
\liminf _{i} g_{i}=\liminf _{i} z_{i}^{*} \leqslant z^{*} \leqslant \lim \sup _{i} z_{i}^{*}=\lim \sup _{i} g_{i} \text { on } X,
$$

and so $\left.z^{*}\right|_{X} \in \operatorname{liminfsup}_{i} g_{i}$. (14.4) now gives $\operatorname{argmax}_{X}\left(\left.y^{*}\right|_{X}-\left.z^{*}\right|_{X}-\rho \psi\right)=\emptyset$, which would contradict (14.2) since $x^{*}:=\left(y^{*}-z^{*}\right) / \rho \in E^{*}$.

The following converse minimax theorem was proved in [7, Theorem 15, p.717]. However, we include in Lemma 18 in the appendix a proof (using Goldstine's theorem) of Lemma 15 that is valid for the special case when $E$ is normed space. 
LemMA 15. Suppose that $X$ is bounded and complete in $E$ and, for all nonempty convex equicontinuous subsets $Y$ of $E^{*}, \inf _{Y} \sup _{X} \delta=\sup _{X} \inf _{Y} \delta$. Then $X$ is $w\left(E, E^{*}\right)$-compact.

If we now combine Theorem 14 and Lemma 15, we obtain the following nonlinear version of James's theorem. (If $Y$ is equicontinuous in $E^{*}$ then $\langle\cdot, \cdot\rangle$ is bounded on $X \times Y$ and, further, $Y$ is $w\left(E^{*}, E\right)$ relatively compact in $E^{*}$, from which (14.1) is satisfied.)

THEOREM 16. Suppose that $X$ is bounded and complete in $E$ and there exists $\psi \in \ell_{\infty}(X)$ such that

$$
\text { for all } x^{*} \in E^{*}, \quad \operatorname{argmax}_{X}\left(\left.x^{*}\right|_{X}-\psi\right) \neq \emptyset .
$$

Then $X$ is $w\left(E, E^{*}\right)$-compact.

REMARK 17. James gave an example in [3] of an incomplete normed space $E$ such that every bounded linear functional on $E$ attains its norm on the unit ball, $X$, of $E$. $X$ clearly satisfied the condition (14.2) with $\psi=0$. However, since $E$ is not reflexive, $X$ is not weakly compact.

\section{APPENDIX}

While the preceding analysis is valid for locally convex spaces, it is possible to give another proof of Lemma 15 for normed spaces that uses more standard techniques. For the convenience of the reader, we give details of this proof in Lemma 18 below.

Lemma 18. Let $E$ be a real normed space and suppose that $X$ is bounded and complete in $E$ and, for all nonempty convex equicontinuous subsets $Y$ of $E^{*}$, $\inf _{Y} \sup _{X} \delta=\sup _{X} \inf _{Y} \delta$. Then $X$ is $w\left(E, E^{*}\right)$-compact.

Proof: Let $~$ stand for the canonical map from $E$ into $E^{* *}$ or the canonical map from $E^{*}$ into $E^{* * *}$, as the case may be. Write $\widetilde{X}$ for the $w\left(E^{* *}, E^{*}\right)$-closure of $\widehat{X}$ in $E^{* *}$. We first prove that

$$
x^{* *} \in \widetilde{X} \text { and } x^{* * *} \in E^{* * *} \quad \Longrightarrow \quad\left\langle x^{* *}, x^{* * *}\right\rangle \leqslant \sup _{x \in X}\left\langle\widehat{x}, x^{* * *}\right\rangle .
$$

To this end, suppose that $x^{* *} \in \tilde{X}$ and $x^{* * *} \in E^{* * *}$. Then

$$
x^{*} \in E^{*} \quad \Longrightarrow \quad\left\langle x^{*}, x^{* *}\right\rangle \leqslant \sup _{x \in X}\left\langle x^{*}, \widehat{x}\right\rangle=\sup _{x \in X}\left\langle x, x^{*}\right\rangle
$$

and Goldstine's theorem (see, for instance, [6, Section 28.40, p.777]), provides us with a bounded net $\left\{x_{\lambda}^{*}\right\}_{\lambda \in \Lambda}$ in $E^{*}$ such that $\widehat{x_{\lambda}^{*}} \rightarrow x^{* * *}$ in $w\left(E^{* * *}, E^{* *}\right)$. Let $\lambda_{0} \in \Lambda$, and $Y=\operatorname{co}\left\{x_{\lambda}^{*}: \lambda \geqslant \lambda_{0}\right\} \subset E^{*}$. Then, from (18.2)

$$
\inf _{\lambda \geqslant \lambda_{0}}\left\langle x^{* *}, \widehat{x_{\lambda}^{*}}\right\rangle=\inf _{\lambda \geqslant \lambda_{0}}\left\langle x_{\lambda}^{*}, x^{* *}\right\rangle=\inf _{x^{*} \in Y}\left\langle x^{*}, x^{* *}\right\rangle \leqslant \inf _{Y} \sup _{X} \delta
$$


Since $Y$ is equicontinuous, by hypothesis,

$$
\begin{aligned}
\inf _{\lambda \geqslant \lambda_{0}}\left\langle x^{* *}, \widehat{x_{\lambda}^{*}}\right\rangle & \leqslant \sup _{X} \inf _{Y} \delta=\sup _{x \in X} \inf _{x^{*} \in Y}\left\langle\widehat{x}, \widehat{x^{*}}\right\rangle \\
& =\sup _{x \in X} \inf _{\lambda \geqslant \lambda_{0}}\left\langle\widehat{x}, \widehat{x_{\lambda}^{*}}\right\rangle \leqslant \sup _{x \in X} \lim _{\lambda}\left\langle\widehat{x}, \widehat{x_{\lambda}^{*}}\right\rangle=\sup _{x \in X}\left\langle\widehat{x}, x^{* * *}\right\rangle .
\end{aligned}
$$

This completes the proof of $(18.1)$, since $\inf _{\lambda \geqslant \lambda_{0}}\left\langle x^{* *}, \widehat{x_{\lambda}^{*}}\right\rangle \rightarrow \lim _{\lambda}\left\langle x^{* *}, \widehat{x_{\lambda}^{*}}\right\rangle=\left\langle x^{* *}, x^{* * *}\right\rangle$ as $\lambda_{0}$ runs through $\Lambda$. Now the canonical map is a norm-isometry from $X$ onto $\widehat{X}$, and so $\widehat{X}$ is norm-closed and (by convexity) also $w\left(E^{* *}, E^{* * *}\right)$-closed. Thus (18.1) implies that $\tilde{X} \subset \widehat{X}$, from which $\widehat{X}=\tilde{X}$. The Banach-Alaoglu theorem now gives us that $\widehat{X}$ is $w\left(E^{* *}, E^{*}\right)$-compact. On the other hand, the canonical map is also a $w\left(E, E^{*}\right)$ $-w\left(E^{* *}, E^{*}\right)$ homeomorphism from $X$ onto $\widehat{X}$, and so $X$ is $w\left(E, E^{*}\right)$-compact, as required.

\section{REFERENCES}

[1] K. Fan, I. Glicksberg and A.J. Hoffman, 'Systems of inequalities involving convex functions', Proc. Amer. Math. Soc. 8 (1957), 617-622.

[2] G. Godefroy, 'Some applications of Simons' inequality', Serdica Math. J. 26 (2000), 59-78.

[3] R.C. James, 'A counterexample for a sup theorem in normed spaces', Israel J. Math. 9 (1971), 511-512.

[4] E. Oja, 'Geometry of Banach spaces having shrinking approximations of the identity', Trans. Amer. Math. Soc. 352 (2000), 2801-2823.

[5] J.D. Pryce, 'Weak compactness in locally convex spaces', Proc. Amer. Math. Soc. 17 (1966), 148-155.

[6] E. Schechter, Handbook of analysis and its foundations (Academic Press, San Diego, 1997).

[7] S. Simons, 'Maximinimax, minimax, and antiminimax theorems and a result of R.C. James', Pacific. J. Math. 40 (1972), 709-718.

[8] S. Simons, 'An eigenvector proof of Fatou's lemma for continuous functions', Math. Intelligencer 17 (1995), 67-70.

[9] S. Simons, Minimax and monotonicity, Lecture Notes in Mathematics 1693 (Springer-Verlag, Berlin, Heidelberg, New York, 1998).

Departamento de Matemática Aplicada

E. U. Arquitectura Técnica

Universidad de Granada

18071 Granada

Spain
Department of Mathematics

University of California

Santa Barbara, CA 93106-3080

United States of America 